\title{
Search for rational ratio of maximum speed and minimum fuel consumption of the vehicle
}

\author{
Maxim Sidorov ${ }^{1}$, Igor Eliseev², Vladimir Sidorov², and Svetlana Golubina ${ }^{2, *}$ \\ ${ }^{1}$ Kaluga Branch of Russian Timiryazev State Agrarian University, Kaluga, Russian Federation \\ ${ }^{2}$ Kaluga Branch of Bauman Moscow State Technical University (National Research University), \\ Kaluga, Russian Federation
}

\begin{abstract}
The gear ratio of the final drive and the distribution of the vehicle mass along the axles has a great influence on the technical and operational parameters of the vehicle. The article discusses the use of mathematical modelling in the MATLAB Simulink and the planning of the experiment to identify the optimal values of the final drive ratio and the mass distribution along the axles when driving a vehicle on a horizontal reference surface. A computational model for determining the moment of resistance on the wheels is developed when the vehicle moves along a supporting surface without slopes. The roughness and unevenness of the surface were not taken into account in the above model. The response function is obtained by studying the dependence of change in two parameters: the speed and fuel consumption of the car from two factors of variation: the final drive ratio and the mass distribution along the axles. The steep ascent method is used to determine the optimal parameters of the vehicle design, which allow achieving maximum speed with minimum fuel consumption when the vehicle is moving on a horizontal supporting surface.
\end{abstract}

\section{Introduction}

The gear ratio of the final drive and the distribution of the vehicle mass along the axles has a great influence on the technical and operational parameters of the vehicle. Thus, the traction properties and the maximum speed of the vehicle depends on the final drive ratio and the weight distribution of the vehicle along the axles determines the handling and traction $[1,2]$. However, the influence of these parameters is double character. Therefore, the search for optimal conditions for achieving maximum speed with minimum fuel consumption is an urgent task.

\section{Purpose of the work}

To determine the optimal values of the gear ratio and the vehicle mass distribution along the axles to achieve a balance between the minimum fuel consumption and the maximum speed of the vehicle.

\footnotetext{
* Corresponding author: asbina@yandex.ru
} 


\section{Methods}

To study the effect of the final drive ratio and the mass distribution on the axles on the change in the speed and fuel consumption of the vehicle, the differential transmission scheme of a vehicle with a rear-driving axle was adopted, shown in Figure 1. [4-6].

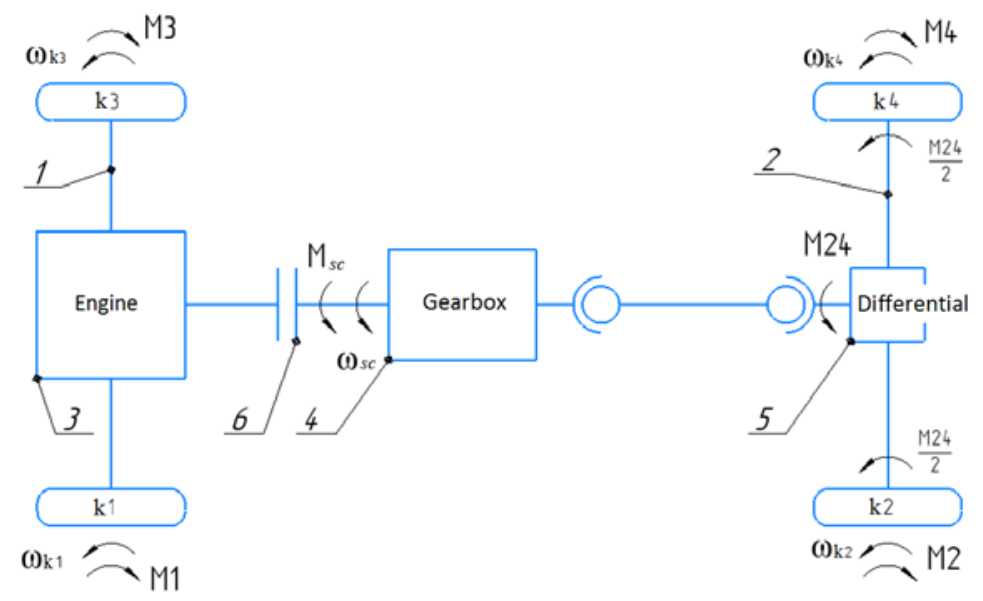

Fig. 1. Differential transmission of a vehicle with a rear-driving axle: 1, 2-front and rear axles of the vehicle; 3 - internal combustion engine; 4 - gearbox; 5 - csymmetrical wheel-to-wheel differential; 6 - clutch; $\mathrm{k} 1$...k 4 - wheel number; $\mathrm{M}_{\mathrm{i}}$-moment of resistance on the i-th wheel; $\mathrm{M}_{24}$ - torque applied to the rear transmission shaft; $\mathrm{M}_{\mathrm{sc}}$ - clutch torque; $\omega_{\mathrm{sc}}$-angular velocity of the driven clutch disc; $\omega_{\mathrm{ki}}$ - angular velocity of rotation of the i-th wheel.

The study model is a system of differential equations [3-6]:

$$
\left\{\begin{array}{l}
J_{k} \cdot \dot{\omega}_{k 1}=-M_{1} \\
J_{k} \cdot \dot{\omega}_{k 2}=0.5 M_{24} \cdot i_{f d}-M_{2} \\
J_{k} \cdot \dot{\omega}_{k 3}=-M_{3} \\
J_{k} \cdot \dot{\omega}_{k 4}=0.5 M_{24} \cdot i_{f d}-M_{4} \\
\dot{\omega}_{s c}=0.5\left(\dot{\omega}_{k 2}+\dot{\omega}_{k 4}\right) i_{f d} \cdot i_{k p} \\
J_{d} \cdot \dot{\omega}_{s c}=M_{s c}-\frac{M_{24}}{i_{k p}}
\end{array}\right.
$$

where $J_{k}, J_{d}$ - moments of inertia of the wheel and engine; $\dot{\omega}_{k i}-$ angular acceleration of the $i$-th wheel; $i_{k p}$ and $i_{f d}$ - gear ratios of gearbox and final drive.

This system of equations is presented in matrix form: $A x=b$, where $x_{1}=\dot{\omega}_{k 1}, x_{2}=\dot{\omega}_{k 2}, x_{3}=\dot{\omega}_{k 3}, x_{4}=\dot{\omega}_{k 4}, x_{5}=\dot{\omega}_{s c}, x_{6}=M_{24}$.

The matrix $A$ has the form: 


$$
A=\left[\begin{array}{cccccc}
J_{k} & 0 & 0 & 0 & 0 & 0 \\
0 & J_{k} & 0 & 0 & 0 & \frac{-i_{f d}}{2} \\
0 & 0 & J_{k} & 0 & 0 & 0 \\
0 & 0 & 0 & J_{k} & 0 & \frac{-i_{f d}}{2} \\
0 & \frac{i_{f d} i_{k p}}{2} & 0 & \frac{i_{f d} i_{k p}}{2} & -1 & 0 \\
0 & 0 & 0 & 0 & J_{d} & \frac{1}{i_{k p}}
\end{array}\right] .
$$

The matrix $b$ has the form:

$$
b=\left[\begin{array}{c}
-M_{1} \\
-M_{1} \\
-M_{1} \\
-M_{1} \\
0 \\
-M_{s c}
\end{array}\right] .
$$

Solving a system of equations: (1):

$$
X=A^{-1} \cdot b .
$$

To solve the system of differential equations in the MATLAB Simulink, the block shown in Figure 2 was used.

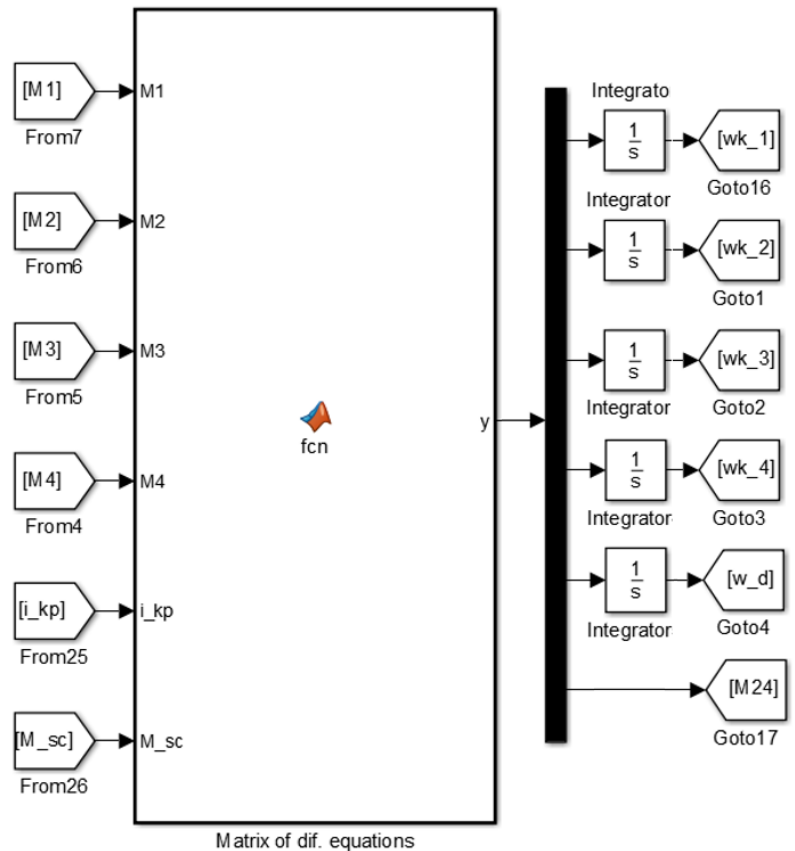

Fig. 2. Block for solving a system of differential equations. 
Input data (designations are given according to the model in the MATLAB Simulink environment):

$M 1, M 2, M 3, M 4$ - moments of resistance to the movement of the vehicle on each wheel; $i \_k p$ - transmission gear ratio; $M \_s c$ - transmission input shaft torque.

The parameters are set as: the moments of inertia of the wheel and the rotating parts of the engine, as well as the gear ratio of the final drive.

Output data (designations are given according to the model in the MATLAB Simulink environment):

$\omega \mathrm{k} \_1, \omega \mathrm{k} \_2, \omega \mathrm{k} \_3, \omega \mathrm{k} \_4$ - angular velocity at each wheel; $\omega \_\mathrm{d}$ - engine crankshaft angular velocity; M24 - torque transmitted from the gearbox to the rear drive wheels.

The maximum vehicle speed is calculated from the condition of the vehicle's movement on a horizontal paved road section. The drag force of the vehicle is composed of the rolling resistance force and the air resistance force. The rolling resistance force of the wheel is determined as follows:

$$
P_{f}=R \cdot \frac{a}{r_{k}}=R \cdot f
$$

where $R$ - normal reaction of the bearing surface; $f=\frac{a}{r_{k}}-$ rolling resistance coefficient.

On a horizontal road, $R=G$ is the weight per wheel.

The rolling resistance coefficient $f$ is determined by the expression:

$$
f=f_{0} \cdot\left(1+\frac{V^{2}}{1500}\right),
$$

where $V$ - wheel speed in $\mathrm{m} / \mathrm{s} ; f_{0}$ - coefficient of rolling resistance at a speed close to zero.

Air resistance force is:

$$
P_{w}=S \cdot V^{2} \cdot k_{w}
$$

where $S$ - frontal area of the vehicle; $V$ - vehicle speed; $k_{w}$ - air resistance coefficient.

The block for determining the moment of resistance on the wheel through the force of resistance to the movement of vehicle is shown in Figure 3. 


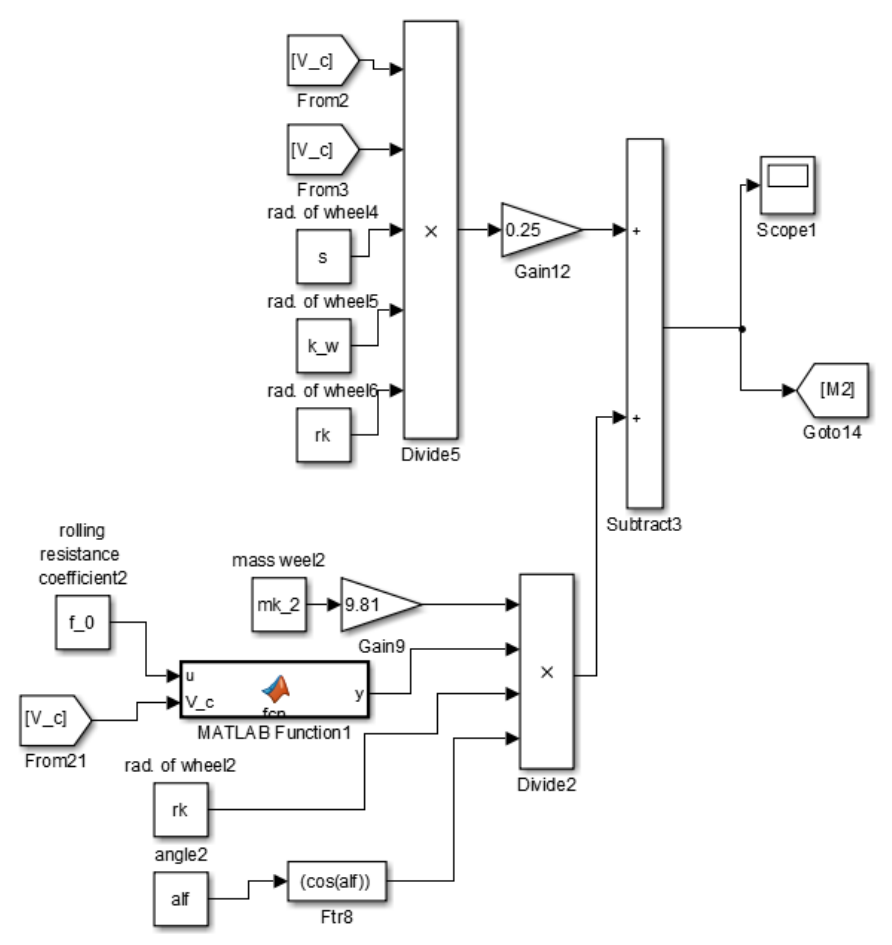

Fig. 3. Block for determining the moment of resistance on a vehicle wheel in MATLAB Simulink.

Engine torque and specific effective fuel consumption are set using an external speed characteristic. The block for determining the torque and specific effective fuel consumption depending on the angular velocity of the crankshaft is shown in Figure 4.

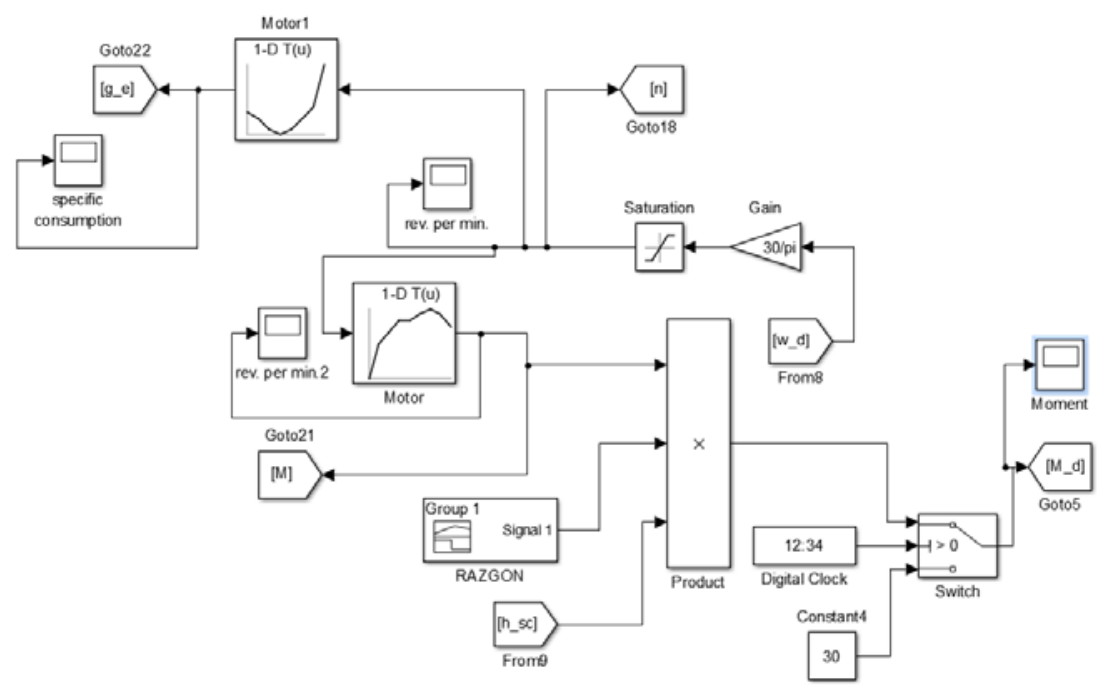

Fig. 4. Block for determining the torque and specific effective fuel consumption of the engine.

In the "Motor" and "Motor1" blocks, the external characteristic of the engine is set in Fig. 5. 


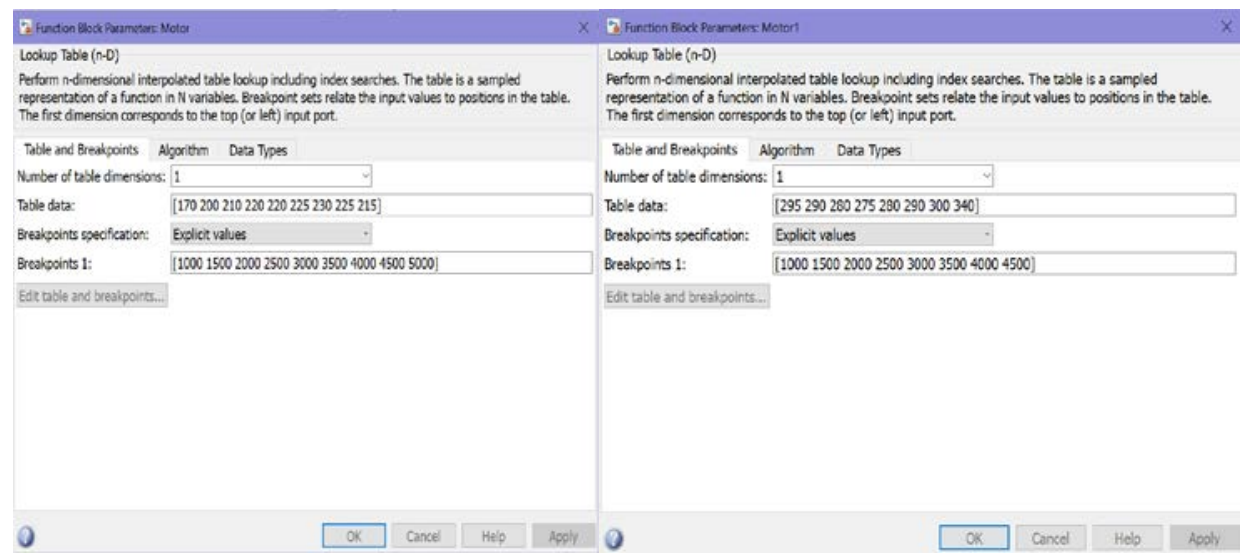

Fig. 5. Setting the values of the external characteristic of the engine.

The volumetric fuel consumption per one hundred kilometers of the vehicle's path is determined by the formula:

$$
Q_{s}=\frac{g_{e} \cdot N_{e}}{36 \cdot V \cdot \rho_{T}},
$$

where $g_{e}$ - specific fuel consumption; $N_{e}$ - engine power; $\rho_{T}$ - density.

The block for determining the volume fuel consumption per one hundred kilometers of the vehicle's path is shown in Figure 6.

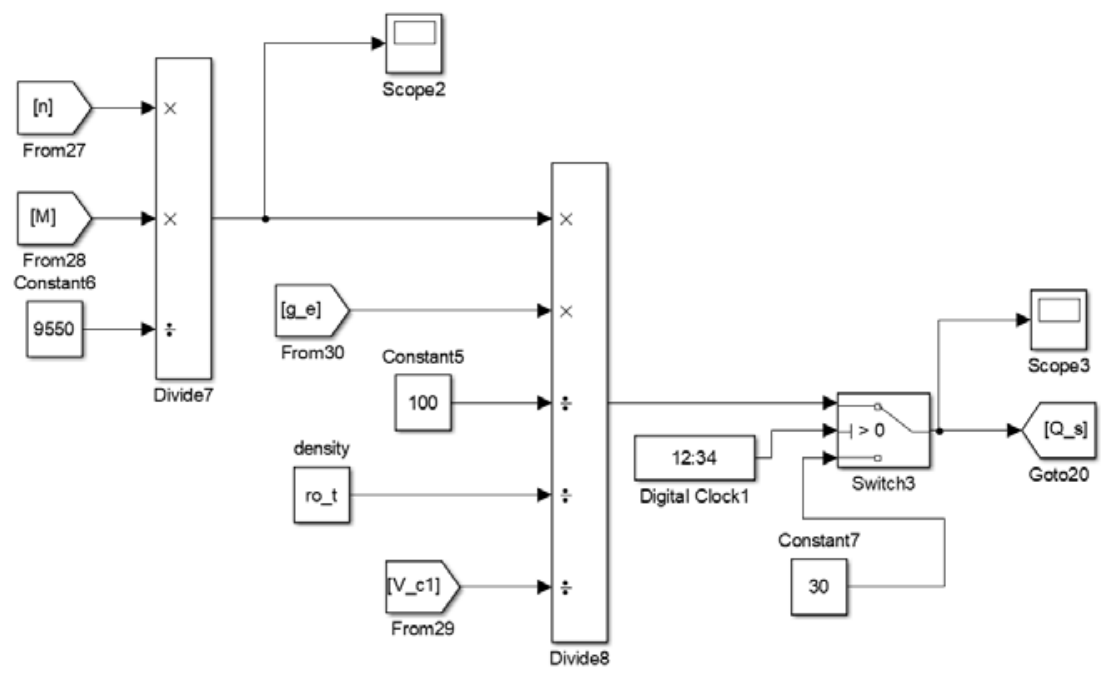

Fig. 6. Block for determining the volume fuel consumption per one hundred kilometers of the vehicle's path.

To study the dependence of the change in two parameters, it is necessary to obtain a function of the form $[7,8]$ :

$$
y\left(x_{1}, x_{2}\right)=a \cdot y_{1}+b \cdot y_{2}
$$


Where $a, b$ - proportionality coefficients that allow considering the interaction of the equations $y_{1}\left(x_{1}, x_{2}\right)$ and $y_{2}\left(x_{1}, x_{2}\right)$ on the same scale; $y_{1}$ - vehicle speed, $\mathrm{km} / \mathrm{h} ; y_{2}-$ fuel consumption, $1 / 100 \mathrm{~km}$.

With two factors, the response function model has the following form [7-8]:

$$
y=b_{0}+b_{1} \cdot x_{1}+b_{2} \cdot x_{2}+b_{12} \cdot x_{1} \cdot x_{2} .
$$

\section{Results}

The limits of variation of the factors are shown in Table 1 and the plan-matrix of experiments in Table 2.

Table 1. Values of levels and intervals of variation of factors.

\begin{tabular}{|c|c|c|c|c|}
\hline \multirow{2}{*}{ Name and symbol of factors } & \multicolumn{3}{|c|}{ Variation levels } & \multirow{2}{*}{ Variation intervals } \\
\cline { 2 - 4 } & -1 & 0 & +1 & \\
\hline Final drive ratio $\left(\mathrm{x}_{1}\right)$ & 3,5 & 4,5 & 5,5 & 1 \\
\hline Rear axle share of vehicle weight $\left(\mathrm{x}_{2}\right)$ & 0,4 & 0,5 & 0,6 & 0,1 \\
\hline
\end{tabular}

Table 2. The plan-matrix of experiment $2^{2}$ for the function $y$.

\begin{tabular}{|c|c|c|c|c|c|}
\hline \multirow{2}{*}{ Experience number } & \multicolumn{2}{|c|}{ Natural values } & \multicolumn{2}{|c|}{ Factors } & \multirow{2}{*}{ Response } \\
\hline & $x_{1}$ & $x_{2}$ & $x_{1}$ & $x_{2}$ & \\
\hline 1 & 5,5 & 0,6 & 1 & 1 & $-14,925$ \\
\hline 2 & 3,5 & 0,6 & -1 & 1 & 29,271 \\
\hline 3 & 5,5 & 0,4 & 1 & -1 & 0,244 \\
\hline 4 & 3,5 & 0,4 & -1 & -1 & 41,371 \\
\hline
\end{tabular}

With two factors, a model of the response function in the form of a regression equation was obtained:

$$
y=-14.925-21.331 \cdot x_{1}-6.817 \cdot x_{2}-0.768 \cdot x_{1} \cdot x_{2} .
$$

The results of the steep ascent are shown in Table 3.

Table 3. Steep ascent on the response surface.

\begin{tabular}{|c|c|c|c|}
\hline step & $x 1$ & $x 2$ & $y$ \\
\hline 0 & 3,5 & 0,6 & 61,602 \\
\hline 1 & 3,58 & 0,592 & 61,6896 \\
\hline 2 & 3,66 & 0,584 & 62,504 \\
\hline 3 & 3,74 & 0,576 & 63,455 \\
\hline 4 & 3,82 & 0,568 & 64,406 \\
\hline 5 & 3,9 & 0,56 & 65,04 \\
\hline 6 & 3,98 & 0,552 & 65,357 \\
\hline 7 & 4,06 & 0,544 & 63,625 \\
\hline 8 & 4,14 & 0,536 & 62,21 \\
\hline 9 & 4,22 & 0,528 & 62,0489 \\
\hline 10 & 4,3 & 0,52 & 61,9754 \\
\hline 11 & 4,38 & 0,512 & 61,9264 \\
\hline 12 & 4,46 & 0,504 & 61,331 \\
\hline 13 & 4,54 & 0,496 & 60,599 \\
\hline 14 & 4,62 & 0,488 & 60,55 \\
\hline 15 & 4,7 & 0,48 & 60,501 \\
\hline 16 & 4,78 & 0,472 & 60,452 \\
\hline 17 & 4,86 & 0,464 & 60,3566 \\
\hline 18 & 4,94 & 0,456 & 59,671 \\
\hline
\end{tabular}


The results of the search for the optimal value by the steep ascent method are presented in the form of a graph of the dependence of the function response from the step number of the movement to the optimal value (Fig. 7)

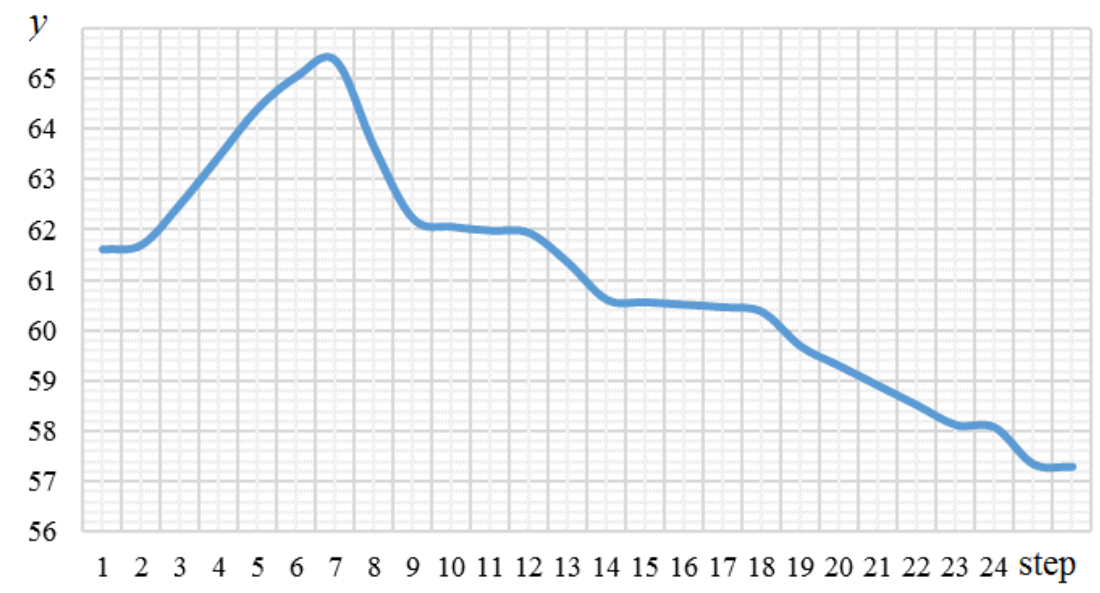

Fig. 7. The response function - the step number $\lambda=0.552$.

As a result, of the search for the optimum by the steep ascent method, it was possible to determine the optimal ratio between the final drive ratio and the mass distribution between the axles. This value corresponds to the calculations in step 6 . The parameters in this case are $i_{f d}=3.98, \lambda=0.552$.

\section{Conclusion}

The developed mathematical model for calculating the speed and fuel consumption of a vehicle allows to study the influence of such parameters as the final drive ratio and the distribution of the mass of the vehicle between the axles, depending on the selected layout scheme.

Based on the planning of the experiment, the optimal values of the final drive ratio and the mass distribution between the axles were determined. The calculation was carried out for the conditions of movement on a horizontal surface. The following optimal values of the gear ratio of final drive $i_{f d}=3.98$ and the distribution of mass between the axles $\lambda=0.552$ were obtained.

\section{References}

1 V.V. Larin, Theory of Wheel Drive (Moscow: Publishing House of BMSTU, 2010)

2. G.M. Kutkov, Tractors and cars. Theory and technological properties (Moscow: Infa-M, 2014)

3. M.M. Zhileykin, G.O. Kotiev, E.B. Sarach, Mathematical models of vehicle systems: methodological guidelines (Moscow: BMSTU, 2018)

4. B.A. Afanasyev, B.N. Belousov, G.I. Gladov and others under the general. Ed. A.A. Polungyan, Design of all-wheel drive wheelers, v. 3 (Moscow: Publishing House BMSTU, 2008) 
5. A.V. Sidorova, P.I. Stepin, V.N. Sidorov, Simulation of oscillations of the center of mass of the wheel machine using the Simulink program, Don Engineering Bulletin, v.4, 6395 (2020)

6. M.V. Sidorov, R.V. Sidorin, Substantiation of the mass of the pickup truck-tractor from the condition of adhesion to the road when changing the lifting angle, International Feasibility Journal, v. 6, p. 50-55 (2020)

7. V.I. Komlatsky, S.V. Loginov, G.V. Komlatsky, Planning and organization of scientific research (Rostov-on-Don: Phoenix, 2014)

8. Yu.P. Adler, E.V. Markova, Yu.V. Granovsky, Experiment planning when searching for optimal conditions (Moscow: Publishing House of Science, 1976) 